\title{
Aleida und Jan Assmann: Kultur als Schrift und Gedächtnis
}

\author{
Andreas Langenohl
}

\section{Allgemeine Einführung}

Die Arbeiten von Aleida und Jan Assmann haben sich für die Kulturwissenschaften im deutschsprachigen Raum vermutlich deshalb als so enorm bedeutsam erwiesen, weil in ihnen die nicht immer stringent begründbaren Trennungen innerhalb der Geisteswissenschaften - zwischen Geschichtsschreibung und Erinnerungsforschung, zwischen Philologie und Archäologie oder zwischen Literatur- und Medienwissenschaft - aufgehoben werden. ${ }^{1}$

Beide Assmanns studierten Ägyptologie und hielten sich zwischen 1968 und 1975 zu Grabungsaufenthalten in Oberägypten auf. Während Jans zweiter Studienschwerpunkt bei der Gräzistik lag, verfolgte Aleida ein anglistisches Studium und promovierte in beiden Fächern 1977 in Heidelberg und Tübingen. Jan Assmann wurde 1976 auf einen ägyptologischen Lehrstuhl in Heidelberg, Aleida 1993 auf einen Lehrstuhl für Anglistik und Allgemeine Literaturwissenschaft in Konstanz berufen. Die Anerkennung, die die Arbeiten der Assmann gefunden haben, zeigt sich in zahlreichen Auszeichnungen (es werden nur einige genannt): Jan erhielt 2006 das Bundesverdienstkreuz Erster Klasse und den Alfred-KruppWissenschaftspreis sowie 2007 den Europäischen Essay-Preis Charles Veillon, Aleida den Forschungspreis für Geisteswissenschaften der Philip-Morris-Stiftung (1999) und den MaxPlanck-Forschungspreis (2009)

Die Zentralbegriffe der Arbeiten der Assmanns sind Gedächtnis, Erinnerung und Schrift. Sie sind an der Rekonstruktion und Systematisierung von Mechanismen kultureller Überlieferung in Europa interessiert und messen darin dem Medium der Schrift herausragende Bedeutung bei. Während Aleida ihren Fokus auf zwei Zeitschwellen legt - die Schwelle von der frühen Neuzeit zur Moderne (16. - 17. Jahrhundert) und den „Zivilisationsbruch“ (Diner 1988) des Holocaust - weitet Jan den zeitlichen Rahmen von Tradierungsprozessen von den frühesten altägyptischen Zeugnissen vor 5600 Jahren bis in die Moderne und die Gegenwart.

Dabei zeichnet die Arbeiten der Assmanns ein relativistischer Konstruktivismus aus, der nicht von einer unbehelligten Zeitreise kultureller Konzepte, sondern von einem Tradierungsmodus ausgeht, der auf intertextuellen Prozessen wie Kommentierung, Kritik und Kontextualisierung beruht (vgl. A. Assmann 1999a: 87; J. Assmann 1999: 30). Dieser Konstruktivismus ruft keine Beliebigkeit der Interpretation aus, sondern arbeitet die Spezifika des kulturell Gewordenen heraus. Er identifiziert den Motor der Rekonstruktion der Vergangenheit für die Gegenwart auf der Ebene der Texte, ihrer Referenzen, Wechseldynamiken und ihrer Konstitution in der Schrift selbst. Natürlich blenden die Assmanns institutionelle Effekte - etwa Machtkämpfe kultureller Eliten, Konflikte um Kanonisierung etc. -

Ich danke Nicole Falkenhayner (Konstanz) und Jürgen Schraten (Gießen) sehr herzlich für wertvolle Kommentare zu diesem Artikel. 
nicht aus, sondern spüren ihnen im Gegenteil mit historischen und soziologischen Mitteln nach (s. v.a. Assmann/Assmann 1987). Dennoch werden solche institutionellen Faktoren erst im Nachgang der genuinen Textlichkeit von Kultur zum Thema. Obwohl das Anthropologische für die Assmanns daher beileibe nicht im Schriftlichen aufgeht, wie sie auch Kultur nicht auf ihre Schriftlichkeit reduzieren (Assmann/Assmann 1983: 268), ist doch der kulturelle Aspekt der condition humaine am deutlichsten und wirkungsvollsten im Prinzip der Schrift und der Literatur, in der die Schrift ihren genuinen Eigenschaften den stärksten Ausdruck verschafft, aufgehoben. Insofern kann die Schrift als „das wichtigste kulturelle Instrument anthropologischer Selbsterkundung" gelten (Assmann/Assmann 2006: 9).

Diese ebenso prägnante wie folgenreiche Einstufung findet sich in der Einleitung des ersten Bandes der Schriftenreihe „Archäologie der literarischen Kommunikation“, die von den Assmanns 1983 mitbegründet wurde. Jan Assmann zufolge ging es den Begründern dieser bis heute existierenden Reihe um eine kulturwissenschaftlich-interdisziplinäre Öffnung von Fragen des literarischen Textes (J. Assmann 1992: 21). Der Schlüssel, der diese Öffnung bewerkstelligen sollte, wurde in den Altertumswissenschaften gesehen. Die Arbeiten Jan Assmanns zum „kulturellen Gedächtnis“ (J. Assmann 1992) sind daher als eine Grundlegung der Kulturwissenschaften aus altertumswissenschaftlicher Perspektive anzusehen, die über die etablierten einzelwissenschaftlichen Positionen wie Literaturwissenschaft, Kommunikationswissenschaft oder Medienwissenschaft hinaus geht. In einigen der frühesten bekannten Hochkulturen nämlich entsteht mit der Schrift ein eigenlogischer Bereich des Kulturellen, der aus den alltäglichen Interaktionszusammenhängen strukturell ausgelagert wird und zu ihnen in ein funktionales Verhältnis, nämlich als „Möglichkeiten externer Zwischenspeicherung" tritt. So erschließt sich die Autonomie des Kulturellen dieses „Außenbereichs der Kommunikation“ (J. Assmann 1992: 22, 23) - aus der Perspektive ihres Konnexes mit der Schrift, den wiederum bevorzugt die Altertumswissenschaften genealogisch herauspräparieren können. Das Altertum ist somit der historische Urgrund der Ausdifferenzierung von „Kultur“, und die Altertumswissenschaften verfügen über die Instrumente, diesen Prozess in seiner paradigmatischen Bedeutung sichtbar zu machen.

Beim zweiten Kontext der Assmannschen Arbeiten handelt es sich um einen intellektuellen Zirkel, der bei der Konstitution von „Archäologie der literarischen Kommunikation“ offensichtlich Pate gestanden hat, nämlich um den 1964 an die Öffentlichkeit tretenden Arbeitskreis „Poetik und Hermeneutik“ (Jauß 1964). Die Kontinuitäten zwischen beiden Schriftreihen zeigen sich auf den ersten Blick in einer Teilnahme von „Poetik“-Mitgliedern an den Tagungen und Publikationen des „Archäologie“-Kreises. ${ }^{2}$ Inhaltlich lässt sich die Agenda von „Archäologie der literarischen Kommunikation“ - Schrift/Kultur und Mensch - vor allem mit zwei wichtigen Impulsen aus dem „Poetik und Hermeneutik“-Umfeld in Verbindung bringen. Zum einen beziehen sich die Assmanns immer wieder auf die Historizität des Verhältnisses von Geschichte und Gedächtnis, die ein zentrales Thema bei Reinhard Koselleck (etwa Koselleck 1989) bildet. Zum anderen findet mit der Rezeptionsästhetik eine zentrale Säule der „Konstanzer Schule“ der Literaturwissenschaft, die von Hans Robert Jauß (1982) und Wolfgang Iser (1972) begründet wurde, Eingang in das kulturwissenschaftliche Programm der Assmanns. ${ }^{3}$ Gemäß den Prämissen der Rezeptionsästhetik

\footnotetext{
2 Diese personalen Kontinuitäten zwischen „Poetik und Hermeneutik“ und „Archäologie der literarischen Kommunikation" zeigen sich beispielhaft in Gumbrecht (1983), Lachmann (1987) und Gumbrecht (1987). Eine der letzten großen Arbeiten Isers (1991) steht in enger inhaltlicher Beziehung zu der an schriftzentrierter Anthropologie interessierten Agenda von „Archäologie der literarischen Kommunikation“.
} 
bildet der literarische Text ein Verweissystem, welches die Aufmerksamkeit des Lesers anleitet und ihm während der Lektüre bestimmte Positionalitäten anbietet. Die Rezeptionsästhetik stellt so neben der Altertumswissenschaft den zweiten Einstiegsort für das kulturwissenschaftliche Programm der Assmanns dar. Sie begründet die kulturelle Zentralität von Schrift und Text in der interaktiven Struktur von Textualität, welche Rezeption, Kommentierung und Kontextualisierung vorwegnimmt und anleitet.

Der Schwerpunkt der vorliegenden Darstellung liegt auf den bei den Assmanns wiederkehrenden und von beiden weiterentwickelten kulturwissenschaftlichen Themen und Theorien. Sie geht zunächst auf jeden der beiden im Einzelnen ein, um am Ende die gemeinsamen Fluchtlinien ihrer Arbeit wieder aufzugreifen.

\section{Jan Assmann: Religion, kulturelles Gedächtnis und die Aktualitäten Ägyptens}

Jan Assmann erweitert die ägyptologische Perspektive auf der Grundlage einer kulturtheoretisch-methodologischen Argumentation zu einer Erinnerungsgeschichte Europas. Nicht nur erschließt er die altägyptische Kultur aus der Sicht ihrer monumentalen Tradierungsleistungen, sondern verfolgt ihre „Gedächtnisspur" (J. Assmann 1998: 1) durch die Jahrtausende bis in die Gegenwart. Der Einfluss der Assmanns auf die gegenwärtige Kulturwissenschaft und vor allem die Erinnerungsforschung wird häufig auf die Trennschärfe ihrer analytischen Kategorien zurückgeführt, allen voran der des „kulturellen Gedächtnisses“ (vgl. Erll 2005, Frank/Rippl 2007). Jedoch führen diese Kategorien Jan Assmann auch inhaltlich auf Gebiete weit jenseits der Ägyptologie, nämlich in Richtung einer „Sinngeschichte" Europas (J. Assmann 1999: 1), der es darum geht, wie sich durch Auseinandersetzungs-, Interpretations- und Kanonisierungsprozesse der ägyptischen Vergangenheit kulturelle Institutionalisierungs- und Konfliktmuster in Europa seit der Antike bildeten, die die Kultur in Europa und darüber hinaus bis heute prägen: „Eine ,Sinngeschichte“ thematisiert Geschichte als kulturelle Form, wobei ihr ereignisgeschichtlicher Ablauf den Hintergrund und die sinnstiftenden und sinnreflektierenden Diskurse den Vordergrund bilden." (J. Assmann 1999: 11)

An zentraler Gelenkstelle dieses dem Gedächtnis Ägyptens durch die Jahrtausende nachspürenden Werks steht das Buch Das kulturelle Gedächtnis (1992), in dem Befunde aus verschiedenen antiken Hochkulturen zu einer Konzeptualisierung des kulturellen Gedächtnisses verdichtet werden. Zur Neuzeit kommt Assmann seit den 1990er Jahren, als er beginnt, sich für die europäische Ägyptenrezeption seit der Renaissance zu interessieren.

\section{Das kulturelle Gedächtnis}

Der Begriff des „,kulturellen Gedächtnisses“ geht, Jan zufolge, auf eine gemeinsame Publikation mit Aleida zurück (J. Assmann 1995a: 9; Assmann/Assmann 1990). Er ist auch in älteren Publikationen der Assmanns anzutreffen (Assmann/Assmann 1987). ${ }^{4}$ Assmann gibt dem Begriff dadurch Kontur, dass er ihn vor dem Hintergrund der Schriften Maurice Halbwachs' (1966 [1925], 1967) dem Begriff des „kommunikativen Gedächtnisses“ gegenüber-

4 Siehe auch die Hinweise in Frank/Rippl (2007, Fn. 30). 
stellt. Das kommunikative Gedächtnis ist an den Halbwachsschen Begriff des „,kollektiven Gedächtnisses" angelehnt, weist jedoch eine andere Nuancierung auf, weil es in erster Linie einen formal beschreibbaren Erinnerungsmodus bezeichnet, während das Halbwachssche kollektive Gedächtnis starke Bezüge zu inhaltlichen Erinnerungsrahmen (etwa familialen, freundschaftlichen etc.) unterhält. Während das kommunikative Gedächtnis sich auf Erinnerungspraktiken bezieht, die im interaktionalen face-to-face-Modus zirkulieren und daher nicht weiter als die Erinnerung der ältesten Gesellschaftsmitglieder zurückreichen, bezeichnet das kulturelle Gedächtnis Repräsentationen der Vergangenheit, die erst jenseits dieser Schwelle einsetzen und, da sie nicht durch persönliche Erinnerungen verbürgbar sind, notwendig institutionelle Gestalt annehmen müssen (J. Assmann 1992: 58) ${ }^{5}$

Das kulturelle Gedächtnis stellt somit ein formatiertes Reservoir semantischer Bestände dar, die in unterschiedlichen Formen gesellschaftlich institutionalisiert sind (vgl. auch J. Assmann 1999: 27). Dies begründet den Assmanschen Aufgriff von Konrad Ehlichs (1983) Begriff der ,zerdehnten Situation“ (etwa in J. Assmann 1995a: 9f.), d.h. eines sich erst in der Überlieferung semantischer Bestände konstituierenden gemeinsamen Sinnhorizonts zwischen Autor und Leser eines Texts, der im Falle der Antike Jahrtausende überspannen kann. Hier findet die Weichenstellung für Jan Assmanns textualen Kulturbegriff statt: Das kulturelle Gedächtnis weist eine textuale Struktur auf, nicht weil es sich bevorzugt in schriftlichen Überlieferungen manifestiert, sondern weil seine Bestände von Rezipienten späterer Zeiten „gelesen“ werden müssen und erst durch diesen Akt der Lektüre Interpretation möglich wird. Kulturelle Weitergabe ist somit eine Lektüre vorgefundener kultureller Artefakte (J. Assmann 1995a: 9-12). Daher manifestiert sich das kulturelle Gedächtnis immer als eine Interpretationsleistung und ist insofern durch die jeweilige Gegenwart des Lesens bestimmt, bleibt aber an die Ausgangstexte durch eine Kette von aufeinander folgenden Lektüren rückgebunden. Kulturgeschichte stellt sich somit als Gedächtnisgeschichte dar.

Jan Assmanns erinnerungstheoretischer Hauptbeitrag wird zuweilen in seiner Verknüpfung des Erinnerungs- mit dem Identitätsbegriff und mit Prozessen politischer Legitimierung erblickt (vgl. Erll 2005). Seine Arbeiten stellen hier indes vor allem Differenzierungshilfen zur Verfügung, denn sie machen deutlich, dass das kulturelle Gedächtnis unterschiedliche Ligaturen mit Identitätsstiftung und politischer Legitimation eingehen kann. Während in Ägypten beispielsweise das kulturelle Gedächtnis der Rechtfertigung von Herrschaft diente, indem es die Kontinuität des Staates zum Ausdruck brachte, ohne eine sich von anderen Kollektiven abgrenzende kollektive Identität der Ägypter zu codieren (J. Assmann 1992: 167-177), diente im antiken Griechenland des 8. Jahrhunderts die Erinnerung an das heroische Zeitalter der symbolischen Konstitution hellenischer Identität, ohne mit der Idee eines politischen Gemeinwesens und politischer Herrschaft verquickt zu sein (J. Assmann 1992: 272-280). Einen Zwischenfall bildet die Exodus-Erinnerung der Israeliten, weil sie einen Erinnerungsimperativ zum Ausdruck bringt, der zwar eindeutig eine kollektive Identität als "Gottes Volk" symbolisierte, nicht aber die Legitimität der politischen Elite begründete (J. Assmann 1992: 202-207). Wenn daher auch Erinnerung, im Sinne einer anthropologischen Konstante, der Ort ist, ,in den die Gesellschaft sich einschreibt mit ihren Ansprüchen und Verpflichtungen" (J. Assmann 1995b: 52), kann daraus nicht gefolgert werden, dass sich durch das kulturelle Gedächtnis immer schon eine politische Identität konstituiert.

5 Vgl. zusammenfassend die Tabelle in J. Assmann (1992: 56). 


\section{Die ägyptische Religion als Paradigma, Vergleichspunkt und Topos}

Die Arbeiten Assmanns zum kulturellen Gedächtnis rahmen seine Studien zum Vergleich antiker „Hochreligionen“. Assmann konzipiert den Religionsvergleich nicht nur als eine Gegenüberstellung in sich ruhender Glaubenssysteme, sondern arbeitet die wechselseitigen Abgrenzungen antiker Religionen und insbesondere die Besonderung der israelitischen/jüdischen Religion, der Götter-Pantheen der antiken Großreiche und später des Christentums in ihren Rezeptionen ägyptischer Religion heraus. Dabei formuliert er Grundsätze einer „kulturwissenschaftlichen Thanatologie“ (J. Assmann 2001: 23) vor allem anhand einer Gegenüberstellung der ägyptischen Religion und der sich von dieser abgrenzenden mosaischen Religion der Frühzeit.

Auf einer ersten Vergleichsebene geht es um religionsspezifische Umgangsweisen mit dem Tod. Hier bildet das alte Ägypten ein Beispiel für eine sich gegen den Tod auflehnende Kultur, insofern die spirituellen Strukturen auf der (sich seit dem Alten Reich popularisierenden) Idee eines Weiterlebens nach dem Tode beruhen. Dies stellt einen Unterschied zur mosaischen Religion dar, weil letztere dem Tod durch die Idee einer Einlösung des menschlichen Lebens in den Nachkommen, also in der Geschichte, begegnete und daher in ihrer Frühzeit das Konzept eines jenseitigen Weiterlebens ablehnte (vgl. J. Assmann 2001: 20-22). Methodologisch zentral ist der Begriff der "sakramentalen Ausdeutung“ (J. Assmann 1977; J. Assmann 1995a: 14-18; J. Assmann 2001: 454-475): Assmann sieht die rituelle Praxis der Jenseits-Pflege als eine Text-Praxis, d.h. in den Texten in Gräbern und den Vorschriften für die Durchführung von Riten erkennt er nicht einfach Repräsentationen spiritueller Deutungsmuster, sondern die rituelle Handlung selbst. Diese Texte „funktionieren", weil eine Performanzbeziehung zwischen dem Text-Akt und dem Jenseits besteht. Insofern erzeugt - nicht nur bezeugt - die Schrift eine in der ägyptischen Gesellschaft allgegenwärtige Auseinandersetzung mit dem individuellen Tod und dem Weiterleben im Jenseits. Durch diesen performativen Schriftbegriff erscheinen auch die monumentalen Gräber als „Werke“, die im Grabherrn einen Autor haben (J. Assmann 2001: 485f.). Auf diese Weise sind „Kunst, Literatur und Grab“ (J. Assmann 2001: 486) Inkarnationen des Prinzips Schrift, das in Ägypten und darüber hinaus ,eines der entscheidenden Motive zur Ausbildung eines kulturellen Gedächtnisses" darstellt (J. Assmann 2001: 487; vgl. auch J. Assmann 1992: 169-174).

Zweitens begreift Assmann die Herausbildung der nachägyptischen antiken Hochkulturen aus der Perspektive ihrer Bezugnahmen auf Ägypten. Im Zentrum steht dabei die Monografie über Moses den Ägypter, in der Assmann die Entstehung monotheistischer Theologien - die israelitische Religion und in ihrem Gefolge das Christentum - als Auseinandersetzungsformen mit antiken, polytheistischen Glaubenssystemen und vor allem ihrem Prototyp, der ägyptischen Religion sieht. ${ }^{6}$ Den den Monotheismus begründenden Akt nennt Assmann die „mosaische Unterscheidung“ (J. Assmann 1998: 17), d.h. die Unterscheidung zwischen wahrem und falschem Glauben, die den antiken Polytheismen fremd war. Durch seinen Ansatz der „Gedächtnisgeschichte“ (J. Assmann 1998: 26) geht Assmann über den bloßen Religionsvergleich hinaus, denn er sieht die ,mosaische Unterscheidung" als Wiederkehr eines kulturell verleugneten Ereignisses der ägyptischen Religions-

6 Assmann stellt sich hier in eine Kontinuität mit der Freud-Rezeption Thomas Manns, mit dem ihn das Interesse an der Entzifferung der Gedächtnisspur Ägyptens in europäischen Diskursen verbindet (vgl. J. Assmann 2000: 185-199). 
geschichte und damit als einen kulturellen Gedächtnisprozess an. Assmann zufolge reinkarniert in der radikalen Abwendung vom ägyptischen Polytheismus, für die die Geschichte des Auszugs aus Ägypten und des Empfangs der Gesetzestafeln in der israelitischen, jüdischen und christlichen Erinnerung einsteht, die Episode der Amarna-Zeit, während der Pharao Amenophis IV. (Echnaton) bis 1338 regierte, eine radikale Abkehr vom ägyptischen Pantheon durch- und an dessen Stelle eine zentrale Sonnengottheit einsetzte. Diese Episode wurde anschließend aus dem expliziten kulturellen Gedächtnis Ägyptens getilgt, kehrte jedoch in der Form wieder, dass jedwede Herausforderung des Götterpantheons strikt mit Bann, Verbot und Verfolgung belegt wurde. Dies erzeugte bei der israelitischen Gegenreligion - bzw. genauer: ihrer erinnernden Repräsentation als „Auszug aus Ägypten“ - ein umso vehementeres Bestehen auf dem, was die ägyptische Hochreligion kategorisch ausschloss: radikalen Monotheismus und Ächtung der „Idolatrie“. Die monotheistische Unterscheidung zwischen wahrer und falscher Religion erscheint so als Aktualisierungsform eines kulturellen Traumas in Ägypten, weil sie implizit auf Echnatons Sonnenkult, der den Polytheismus radikal unterband, affirmativ Bezug nimmt und erst vor diesem Hintergrund ihre geschichtsmächtige Gestalt gewinnt.

\section{Die Sinngeschichte Ägyptens in Europa}

Mit der Argumentationsfigur, dass erinnerungskulturelle Kontinuitäten durch frühere Verleugnungsprozesse gestiftet werden können, ist der dritte wichtige Aspekt von Assmanns Arbeit angesprochen: der der "Sinngeschichte“ Ägyptens (J. Assmann 1999: 1). Empirischer Ausgangspunkt ist wiederum die Repräsentation Ägyptens in zeitgenössischen Hochkulturen, bei den Israeliten, den Hethitern, bei den Griechen und Römern, dann auch im frühen Christentum und seit der Aufklärung. Gedächtnisgeschichte ist nicht nur mehr als Ereignisgeschichte, sondern auch mehr als Rezeptionsgeschichte, weil Assmann auch auf Deutungsprozesse und -konflikte zu sprechen kommt, die nicht immer explizite Bezugnahmen auf Ägypten aufweisen, von denen aus aber in Ägypten angestoßene kulturelle Sinngebungsprozesse rekonstruierbar sind.

Die Figur des Moses ist ein Beispiel für eine immer neue Aktualisierung des kulturellen Gedächtnisses und hat die Auseinandersetzung mit dem Monotheismus in Europa entscheidend vorgeprägt. Sie wird in einer in der frühen Neuzeit einsetzenden Debatte über die Religion Ägyptens erneut aufgerufen. Nach dem Ende der mittelalterlichen Abwendung von Ägypten als einer heidnischen Gesellschaft gewinnt eine Rezeption der Moses-Geschichte an Boden, die die These wagt, dass der Auszug aus Ägypten nicht für eine $A b$ wendung vom Polytheismus als falscher Religion, sondern für eine emphatische Hinwendung zur eigentlichen ägyptischen Hochreligion stehe. „Die Agenda der Debatte über Moses und Ägypten war [...] die Aufhebung der Mosaischen Unterscheidung im Zeichen von Natur, Vernunft und Toleranz." (J. Assmann 1998: 217) Seit Freuds Arbeiten, vor allem der Studie über den „Mann Moses“ (Freud 1999 [1939]), ist diese Debatte, Assmann zufolge, erstmals in der Lage, das dem exklusorischen Monotheismus zugrunde liegende Trauma kulturell aufzuarbeiten und eine "Versöhnung und Aufhebung der Mosaischen Unterscheidung“" anzusteuern. In dieses Projekt stellt Assmann auch die moderne Ägyptologie und damit seine eigenen Arbeiten ein (vgl. J. Assmann 1998: 240, 42f.). Der dieses Projekt vorbereitenden Phase der europäischen Aufklärung widmet Assmann eine Monografie über Wolf- 
gang Amadeus Mozarts Oper Die Zauberflöte, die er nicht nur, wie es zumeist geschieht, als Darstellung eines Initiationsrituals, sondern als Performanz eines solchen Rituals sieht. Die ägyptisierenden Motive der Oper sind nicht als historische Repräsentationen, sondern als Aktualisierungsformen eines aufklärerischen Potenzials zu sehen, das das 18. Jahrhundert in Ägypten und besonders in der „Isis-Religion“ erblickte (vgl. J. Assmann 2005: 92f.). Die Oper wird daher als eine aufklärerische Aktualisierungsform der Isis-Religion gesehen: die Initiation befreit von personifizierenden Gottesbegriffen und gibt den Blick auf die Natur als Inkarnation der höchsten Vernunft, verkörpert durch Isis, frei.

\section{Aleida Assmann: Differenzierungsformen des kulturellen Gedächtnisses in der Moderne}

Kulturelle Korrelationen der funktionalen Differenzierung der modernen Gesellschaft bilden den Bezugspunkt vieler von Aleida Assmanns Arbeiten. Während Jan die historische Kontingenz der Verbindung zwischen Gedächtnis und Identität betont, stellt diese Verbindung für Aleida den Ausgangspunkt ihres der ästhetischen, politischen, kulturellen und gesellschaftlichen Moderne gewidmeten Werkes dar - einen Ausgangspunkt, den sie zeitlich in exemplarischer Weise in Shakespeares Werk, d.h. an der Schwelle zur Neuzeit verortet sieht (A. Assmann 1999b: 62-83). Je mehr die Kontinuität zwischen Vergangenheit und Gegenwart brüchig wird bzw. zunehmend als reine Kontiguität erscheint, in der historische Perioden aneinander stoßen, ohne inhaltlich aufeinander abbildbar zu sein, „desto enger und dichter wird die Struktur des kulturellen Gedächtnisses. [...] [Traditionen] werden nicht nur von den Herrschenden erfunden, um Machtansprüche symbolisch zu legitimieren und Modernisierungsverluste zu kompensieren, sondern auch von unterdrückten Minderheiten, um über eine gemeinsame Symbolwelt politische Stimme und Handlungsmacht zu gewinnen.“ (A. Assmann 1999a: 89f.) Gerade im Verlust der Vorstellung eines Ineinanderfließens der Epochen liegt die Möglichkeit expliziter Bezugnahmen auf die Vergangenheit. Identitäre Selbstverständigungspraktiken in modernen Gesellschaften sind daher notwendig aus der Perspektive des Gedächtnisses zu erschließen. ${ }^{7}$

Im Unterschied zu Jan Assmanns Konzentration auf Ägypten bzw. dessen Sinngeschichte greift Aleida Assmann immer wieder unterschiedlichste empirische Manifestationsformen des Erinnerns und des Gedächtnisses in Antike, früher Neuzeit und Moderne auf (s. v.a. A. Assmann 1991, A. Assmann 1999b und A. Assmann 2006). Obwohl die Literatur ihr Ausgangs- und privilegierter Bezugspunkt ist, liegen zahlreiche Arbeiten von ihr vor, die sich mit so unterschiedlichen Ausdrucksformen des kulturellen Gedächtnisses wie Denkmälern, Museen, Ritualen und dem Internet befassen.

Dies macht in exemplarischer Weise Aleida Assmanns kleine Monografie zur Entwicklung des Bildungsbegriffs im deutschsprachigen Raum seit dem 18. Jahrhundert deutlich. „Bildung“ ist nicht nur ein geschichtliches, so in keiner anderen Kultur anzutreffendes Konzept, sondern auch ein Erinnerungsmodus, der eine identitäre Aufstiegs- und Verfallsgeschichte codiert und dem Formen der Bewältigung kultureller Orientierungskonflikte eingeschrieben sind (A. Assmann 1993: 111). 
Aleida Assmann trifft in der Literatur und im gelehrten Schrifttum der frühen Neuzeit eine Konstellation gesellschaftsstruktureller und kultureller Differenzierung an, die den kulturellen Reproduktionsmodus der Gesellschaft von Grund auf verändert. Nicht schon mit Erfindung des Buchdrucks, sondern erst mit der Kristallisierung der Konstellation von Autor, Verlag und (anonymem) Publikum entsteht die Möglichkeit des literarischen, fiktionalen Textes im modernen Sinne, weil erst jetzt die literarische Imagination sich mit der Adressierung von Kollektivsubjekten, den sich erfindenden Nationen, verbindet (vgl. A. Assmann 1980: 141f.; A. Assmann 1995: 356; A. Assmann 1999b: 79; A. Assmann 1999a: 130). Wenn daher gerade auch die Neuzeit und Moderne durch zahlreiche Rückgriffe auf die Antike gekennzeichnet sind, ist doch das Neue an ihr das Interesse am Neuen per se, womit sich eine radikale Verzeitlichung und Selbstkontextualisierung kultureller Artefakte - allen voran der Literatur - Bahn bricht (A. Assmann 1999a: 50): ${ }^{8}$ „Die Epochenschwelle der Neuzeit wird $[\ldots]$ charakterisiert als Ablösung eines Wirklichkeitsbegriffs, der eine transzendent garantierte Realität voraussetzt, durch einen anderen, der den Realitätsausweis vom Kriterium des Kontextes abhängig macht. [...] In formaler Analogie zu einem Wirklichkeitsbegriff, der sich in Kontexten manifestiert, steht ein Fiktionsbegriff, der sich als konstitutiver Weltentwurf versteht." (A. Assmann 1980: 107) Gleichzeitig ist diese Epochenschwelle deswegen von Bedeutung, weil sich erst in der Moderne eine quasinatürliche Verbindung zwischen Erinnerung und kollektiver Identität im Sinne Benedict Andersons „vorgestellter Gemeinschaft“ (1987) herstellt (vgl. A. Assmann 1999b: 18f.). Erst in der Neuzeit „,ührte die Frage nach der Erinnerung ins Mark politischer Motivation und politischer Identitätsbildung.“ (A. Assmann 1999b: 83).

Die Beziehung zwischen Erinnerung als anthropologischer Konstante und der Neuzeit bzw. Moderne ist somit mehrschichtig. Einerseits verändern sich Rahmenbedingungen des kulturellen Gedächtnisses in Richtung zunehmender Pluralisierung, Kontingenzsteigerung und Differenzierung (s.u.). Andererseits verschaffen sich in der Moderne allgemeine Merkmale des kulturellen Gedächtnisses und erinnerungskulturelle Tendenzen verstärkt Ausdruck. Das beste Beispiel und Dreh- und Angelpunkt der Argumentation ist auch bei Aleida der Begriff der Schrift: „Das Potential der Schrift besteht in der Kodierung und Speicherung von Informationen jenseits lebendiger Träger und unabhängig von der Aktualisierung in kollektiven Inszenierungen. Das Problem der Schrift besteht in der tendenziell unbeschränkten Akkumulation von Informationen.“ (A. Assmann 1999b: 137) Beides, Potenzial und Problem, verweist auf generelle Problematiken des Erinnerns, die aber erst in der Moderne - mit der Zunahme von Schriftwerken, dem Anschwellen der Archive und zuletzt der Erfindung der „elektronischen Schrift“ des Hypertexts (A. Assmann 1999b: 212) - zum vollen Durchbruch gelangen.

Die Schrift ist es auch, anhand derer Aleida ihre grundlegende und wichtigste kategoriale Differenzierung einführt, nämlich die zwischen „Funktionsgedächtnis“ und „Speichergedächtnis", zu der sie durch eine kritische Auseinandersetzung insbesondere mit Halb-

8 Ich nenne hier nur ein von Assmann angeführtes literarisches Beispiel, nämlich Marcel Prousts $\dot{A}$ la recherche du temps perdu. Assmann argumentiert, dass das literarische Projekt der Verlorenen Zeit auf der Diagnose eines fundamentalen Wirklichkeitsverlusts (verstanden als absolute, unkontextualisierte, ,authentische“ Wirklichkeit) beruhe, angesichts dessen es sich Proust die „Rettung, [die] Bergung und Restitution verlorener Existenz" zur literarischen Aufgabe gemacht habe (A. Assmann 1999a: 54). 
wachs $(1966,1967)$ und Pierre Nora (1989) gelangt. Während sich Halbwachs' Gedächtnistheorie ausschließlich auf Erinnerungsfunktionen, nicht aber auf Erinnerungsbestände beziehe, löse Nora das Gedächtnis von der konkreten Gruppe und übertrage es - als Geschichte - auf vorgestellte Gemeinschaften. Auf dieser Grundlage unterscheidet Assmann zwischen „,bewohntem“ und „unbewohntem Gedächtnis“ und lässt hierüber die Unterscheidung zwischen Gedächtnis und Geschichte in die zwischen Funktions- und Speichergedächtnis münden:

„Das bewohnte Gedächtnis wollen wir das Funktionsgedächtnis nennen. Seine wichtigsten Merkmale sind Gruppenbezug, Selektivität, Wertbindung und Zukunftsorientierung. Die historischen Wissenschaften sind demgegenüber ein Gedächtnis zweiter Ordnung, ein Gedächtnis der Gedächtnisse, das in sich aufnimmt, was seinen vitalen Bezug zur Gegenwart verloren hat. Dieses Gedächtnis der Gedächtnisse schlage ich vor, Speichergedächtnis zu nennen.“ (A. Assmann 1999b: 134)

Die Unterscheidung zwischen Speicher- und Funktionsgedächtnis ist der Ausgangspunkt für weitere Differenzierungen. Die unterschiedlichen Kollektivitätsdimensionen des Erinnerns spricht Aleida Assmann zunächst durch die Unterscheidung zwischen geformten und ungeformten Gedächtnis-“Inhalten“ an: während das „kollektive“ Gedächtnis eine Formung aufweist (etwa eine Zuspitzung auf das Gedächtnis der Nation), ist das ,soziale“ Gedächtnis mit dem „kommunikativen“ Gedächtnis Jan Assmanns darin identisch, dass seine Inhalte keiner stringenten Strukturierung unterliegen, sondern zwischen Individuen und Gruppen flottieren (vgl. A. Assmann 2006: 28-40). Besondere Aufmerksamkeit widmet Aleida Assmann dem kollektiven Gedächtnis der Nation. Einerseits herrscht eine Dialektik zwischen Modernisierung und Mythisierung, „Zwischen der leeren Abstraktion eines modernen funktionalistischen Gebildes [des Staates] einerseits und dem Drang zur Mythisierung dieses Gebildes [durch die nationale Idee] andererseits" (A. Assmann 2006: 41). Andererseits grenzt sich das nationale Gedächtnis gegen die seit dem 18. Jahrhundert entstehende Geschichtsschreibung ab, indem es die Belange der Nation nicht historisiert, sondern als Gedächtnis mythisiert (vgl. A. Assmann 2006: 44). Die Unterscheidung zwischen Geschichte und Gedächtnis steht indes nach dem Holocaust, wie Geschichtskonzepte überhaupt (vgl. etwa Diner 1988, LaCapra 2004), wieder in Frage (s.u.).

\section{Medien des Kulturellen}

Insofern Erinnerung und Gedächtnis sich zu Kultur in einem paradigmatischen Verhältnis befinden, erscheinen Gedächtnismedien als Medien des Kulturellen. Gleichzeitig existieren Gedächtnismedien nach Assmann nicht außerhalb ihrer Interpretation, d.h. Medialität und Metaphorik der Erinnerung sind nicht getrennt voneinander zu sehen (vgl. A. Assmann 1999b: 149-151). In diesem Argument entfaltet sich Aleidas Variante der Assmannschen Schriftzentriertheit: „Schrift“ ist nicht nur das Gedächtnismedium seit alters her, sondern auch eine bevorzugte Gedächtnismetapher und seit der Moderne auch der Erfahrungshorizont für das Scheitern von Gedächtnismedien und -metaphern. Mit der (an die aufkommende Massenliteratur geknüpften) Einsicht seit dem 18. Jahrhundert, dass Texte nicht notwendigerweise und kraft ihrer Schriftlichkeit überleben, sondern größtenteils vergessen werden, „wird das Gedächtnis nun im Rahmen des historischen Bewußtseins von der Tilgung, der 
Zerstörung, der Lücke, dem Vergessen her bestimmt.“ (A. Assmann 1999b: 208) Schrift ist somit wichtigstes Gedächtnismedium wie auch zentrale Gedächtnis- und Kulturmetapher, aber nicht länger, weil ihre Erinnerungskraft als absolut erscheint, sondern weil die Schrift Schwächen zu erkennen gibt, die das Bewusstsein der Moderne insgesamt prägen.

Die anderen Erinnerungsmedien und -metaphoriken bestimmt Assmann unter dem Aspekt, die Problematik der Schrift als Gedächtnismedium und -metapher auszufächern. Hier soll ein Hinweis auf die Körpermetaphorik genügen, die Assmann zufolge einen differenzierten Zugang zum Verhältnis zwischen Konstruktion und Materialität der Vergangenheit bietet. Aleida lehnt, übrigens ebenso wie Jan, ${ }^{9}$ das berühmte Diktum Nietzsches - „Nur was nicht aufhört, wehzutun, bleibt im Gedächtnis" - mit dem Hinweis darauf ab, dass eine auf dem Körper eingeschriebene Vergangenheit - etwa Folter - durch den Prozess der Traumatisierung gerade verleugnet werde. Andererseits weise derselbe Prozess auf Überstände aus der Vergangenheit, die durch einen ausschließlich gegenwartsbezogenen Konstruktivismus nicht adäquat zu fassen seien (vgl. A. Assmann 1999b: 245-250).

\section{Das kulturelle Gedächtnis nach dem Holocaust}

Nach dem Holocaust erfährt das Verhältnis von Geschichte, Gedächtnis und Kultur einen weiteren Differenzierungs- und Problematisierungsschub. Angesichts der Shoah wird es schwierig, sich auf klassisch-moderne Formen des Gedächtnisses, wie etwa das Nationalgedächtnis, zu beziehen. Stattdessen kommen Aporien von Erinnern und Gedächtnis zum Vorschein, die in der Dualität von Erinnern und Vergessen schon immer angelegt waren, nun jedoch eine Zuspitzung erfahren. Der Zivilisationsbruch Nazi-Deutschlands verstärkt zwar das Bewusstsein der Unmöglichkeit eines naiven Bezugs auf Tradition und verstärkt damit eine allgemeine Tendenz der Moderne, nämlich die Kontingentisierung von Kultur; doch ist Tradition damit keineswegs verabschiedet: „Nachdem von Tradition im Sinne einer absichtlichen Weitergabe von Werten und Lebensformen nicht mehr die Rede sein kann, wird die andere Bedeutung von Tradition immer wichtiger: als unbewußte Weitergabe von verdrängter und unbewußter Schuld." (A. Assmann 1999a: 106). Der Begriff des „negativen Gedächtnisses“, den Assmann als Chiffre der in dieser Richtung zugespitzten Situation nach dem Holocaust einführt, betont dementsprechend die „Unfreiwilligkeit und Unverfügbarkeit im Umgang der Betroffenen und Nachgeborenen mit der traumatischen Vergangenheit“ (A. Assmann 2006: 16). Dabei geht es nicht um die These vom ,negativistischen" Gedächtnis (Wingert 1998), in dem sich Kollektive anhand dessen erinnern und identitär definieren, was sie einander nie wieder antun wollen, sondern um eine Perspektive, die den unberechenbaren Wiedereintritt der Vergangenheit in hochmoderne, nachtraumatische Gesellschaften und Kulturen zum Ausgangspunkt nimmt.

Zunächst löst sich die Trennlinie zwischen Gedächtnis und Geschichte auf, da Historiker zunehmend gewahr werden, dass eine neutrale Darstellung des Holocaust ohne Bezug auf die Erzählungen der Überlebenden zu einer „Abstraktion“ verkommt, die die geschichtliche Bedeutung des Ereignisses verfehlt. Insofern also identitätskonkrete Darstellungen der

Seine Ablehnung begründet sich auf der Ignorierung genuin kultureller Tradierungsmechanismen, die Nietzsche ebenso wie Freud ausschließlich in der Metaphorik des Körperlichen und Leibesunmittelbaren gefasst habe: „Auch hier ist ein Reduktionismus am Werk, der die Dynamik auch der kollektiven und kulturellen Erinnerung partout auf die Körpergrenzen des Individuums beschränken will.“ (J. Assmann 2000: 16) 
Vergangenheit in ihre Repräsentation einfließen müssen, wird Geschichte mit Aspekten des Gedächtnisses aufgeladen (vgl. A. Assmann 2006: 44-50). Sodann jedoch werden auch überkommene Modi des kollektiven (politischen, nationalen) Gedächtnisses fraglich, und zwar gerade in ihren identitätsgenerativen Aspekten. Reichert sich Geschichte zunehmend mit Gedächtnis an, verliert letzteres gleichzeitig seinen unproblematischen Identitätsbezug. Dies hängt, Assmann zu Folge, hauptsächlich damit zusammen, dass die überkommene, auch die Moderne prägende Beziehung zwischen Siegern und Verlierern angesichts der Makroverbrechen des 20. Jahrhunderts durch die zwischen Tätern und Opfern herausgefordert wird: während zwischen Siegern und Verlieren zumindest potenziell dasselbe ethischmoralische Koordinatensystem greife und ihre Beziehung reziprok und grundsätzlich ebenengleich sei, sei beides im Verhältnis zwischen Opfern und Tätern nicht der Fall. Der Begriff der „Vergangenheitsbewältigung“ könne sich somit einzig auf das Aus-der-WeltSchaffen von trennenden Erinnerungen zwischen Siegern und Verlierern beziehen, während zwischen Opfern und Tätern eine solches auf Gleichheit beruhendes Vergessen unmöglich sei (vgl. A. Assmann 2006: 67-76); möglich sei nur eine Tätern und Opfern gemeinsame „Vergangenheitsbewahrung" (A. Assmann 2006: 108). Unter diesen Bedingungen diskreditierten sich positive, identitär besetzte Bezüge auf die Vergangenheit, das Kernmerkmal des kollektiven, nationalen Gedächtnisses der Moderne, nachhaltig. Die Täter könnten sich nicht positiv auf die Vergangenheit beziehen, ohne sich dem Verdacht des Verschweigens ihrer Verbrechen auszusetzen, und die Opfer könnten das ihnen widerfahrene Unrecht nicht zum Ausgangspunkt einer positiven Selbstbeschreibung machen, ohne Gefahr zu laufen, in einen exklusorischen Alleinanspruch auf den Opferstatus zu verfallen (vgl. A. Assmann 2006: 77-81).

Schließlich wird der Begriff des Zeugnisses und des Zeugen seit dem Holocaust problematisch (vgl. A. Assmann 2007; vgl. auch Baer 2000). Die (Un-)Möglichkeit, von Auschwitz Zeugnis abzulegen, wirft ein Schlaglicht auf Besonderheiten des Erinnerns nach dem Ende triumphalistischer Gedächtnisrahmen. Zunächst ist Zeugenschaft immer schon mit Sprache und Schrift verbunden, insofern nur derjenige zum Zeugen wird, der einen Bericht gibt. Dann aber begründet sich „moralische Zeugenschaft“ zusätzlich in einem Involviertsein in die berichteten Ereignisse. Aus diesem Grund kann Zeugenschaft von Makroverbrechen nicht an einem objektivistischen Wahrheitsanspruch gemessen werden, der in einen Bericht einmünden würde - vielmehr verkörpert der Zeuge eine Wahrheit, die nicht zu Ende ausgedeutet oder auch nur ausdeutbar ist. Aus diesem Grund - historische „Wahrheit" löst sich von expliziten Berichten und ist nur noch in „Spuren“ aufzusuchen - wächst dem Paradigma des Traumas eine entscheidende Bedeutung zu, weil die Wahrheit des Traumas gerade darin besteht, nicht aussprechbar zu sein (vgl. A. Assmann 2006: 86-103).

\section{Die Modellierung historischer Tiefe aus kulturwissenschaftlicher Perspektive}

Das Forschungsprogramm der Assmanns grenzt sich von einem Präsentismus ab, der etwa in der soziologischen Erinnerungsforschung auf Halbwachs zurückgeführt wird, Erinnerung einzig in ihrer gegenwärtigen Funktionalität in den Blick nimmt und dabei die Vergangenheit aus dem Blick verliert (vgl. Luhmann 1997: 588). Ebenso lehnen sie aber eine Fixierung auf das Speichergedächtnis ab, die Probleme hätte darzutun, auf welche Weise die Vergangenheit der Gegenwart zugänglich gemacht und interpretierend gerahmt wird. Das 
Verhältnis zwischen Vergangenheit und Gegenwart und der Zugang zur Modellierung historischer Tiefe sind bei den Assmanns dialogischer entworfen. Dass Erinnerung stets unter Bedingungen der Gegenwart stattfindet, besagt keineswegs, dass es ausschließlich diese Bedingungen sind, die Erinnerungsprozesse konstituieren. Hinzu kommen drei weitere Faktoren, die mit der Vergangenheit selbst zu tun haben. Erstens finden je gegenwärtige Erinnerungspraktiken immer schon die Artefakte der Vergangenheit vor, deren Vorhandensein in der Gegenwart eine Folge früherer Archivierungsprozesse ist. ${ }^{10}$ Was nicht im Speichergedächtnis anzutreffen ist, kann nicht aktiviert werden. Zweitens stehen Erinnerungspraktiken in einer Geschichte von Deutungen und Interpretationen der Vergangenheit, zu denen sie sich verhalten müssen. Dieses Argumentation nimmt auf Hans Blumenbergs Begriff des „Mythos“ Bezug (Blumenberg 1979; vgl. J. Assmann 1992: 40): die Kontinuierung mythischer Inhalte beruht nicht auf einer unverfälschten Zeitreise der Konzepte und Mytheme, sondern auf einer Kette von Aktualisierungen, die sich Zugang zur "Substanz“ des Mythos durch die Abarbeitung an früheren seiner Aktualisierung verschaffen. Das kulturelle Gedächtnis erschließt historische Tiefe daher auf reflexivem Wege, da es auf frühere Erinnerungs- und Vergessensleistungen bezogen ist und über diese Bande das zu Erinnernde anspielt (vgl. hierzu auch Langenohl 2005). Beispielhaft gelangt dies in Prozessen kultureller Kanonisierung zur Anschauung: da sich der Kanon durch die Affirmation einer bestimmten Deutung des Vergangenen auszeichnet, verneint er alternative Deutungsmöglichkeiten ebenso wie die Möglichkeit, die Vergangenheit dem Vergessen anheim zu geben (vgl. Assmann/Assmann 1987). Drittens bildet die Kette von Interpretationen und reflexiven Aktualisierungen der Vergangenheit einen „Entfaltungsprozeß“ (A. Assmann 1999a: 17) von Gedächtnisinhalten bzw. aus der Vergangenheit überkommenen Artefakten. Aus der Perspektive der Entfaltung von Bedeutung sind es daher nicht nur gegenwärtige Erinnerungsleistungen, die die Vergangenheit definieren, denn die Sinnvalenz des Vergangenen, die ihm immer schon innewohnte, kommt in seinen Aktualisierungen immer aufs Neue zur Kristallisation. Dieses Argument unterscheidet den Assmannschen Konstruktivismus von einem Interpretationsvoluntarismus: wenn auch die Entscheidung darüber, was und wie erinnert wird, in der Gegenwart fällt, kanalisiert das Erinnerte kraft der ihm innewohnenden semiotischen Valenz die Art und Weise, wie es angeeignet und aktualisiert werden kann.

Ein Beispiel hierfür ist das Interesse Europas an den Hieroglyphen (vgl. Assmann/Assmann 2003). Jan Assmann stellt heraus, dass Hieroglyphen Schriftzeichen sind, die sich auf unterschiedliche Weise auf das durch sie Bezeichnete beziehen können (vgl. J. Assmann 1992: 174; Assmann/Assmann 2003: 12). Sie konnten erstens als eine phonetische Konsonantenschrift - semitischen Schriften vergleichbar - gelesen werden, zweitens als eine Silbenschrift und drittens als eine ikonische Schrift, die auf mentale Konzepte unabhängig von ihrer einzelsprachlichen Repräsentation hinwies. Der europäische Zugriff auf die Hieroglyphen erfolgt seit der griechischen Antike in Phasen, in denen jeweils einzelne dieser Bezeichnungsmodi in den Vordergrund gerückt wurden - zuletzt, die Disziplin der Ägyptologie in philologischem Geiste begründend, der phonetische Modus. Damit wurde ein sehr allgemeines Problem menschlicher Kommunikations- und Zeichensysteme immer

10 Dabei ist es zunächst nebensächlich, ob diese Archivierungen natürlichen Ursprung sind (wie die Ruinen und Leichname Pompejis, die durch die sie begrabende Lava „archiviert" wurden) oder auf menschliches Handeln zurückgehen (wie bei der Entscheidung, bestimmte Artefakte in Archiven aufzubewahren und andere dem Zerfall preiszugeben). 
wieder neu verhandelt: „das Verhältnis von Schrift, Sprache, Denken und Wirklichkeit und näherhin die Frage der Möglichkeit motivierter, nichtkonventioneller und in diesem Sinne ,natürlicher' Zeichen“" (Assmann/Assmann 2003: 11). Die Hieroglyphen - und Ägypten als Bestandteil des kulturellen Gedächtnisses Europas - fanden so unterschiedliche Wege ins gesellschaftliche Funktionsgedächtnis, aber dies nicht nur aufgrund je gegenwärtiger Konjunkturen, sondern gerade auch kraft der zeichenanthropologischen Valenz der Hieroglyphen und ihrer mehrschichtigen semiotischen Struktur.

Dies stellt eine Absage an ein lineares Tradierungsmodell dar und favorisiert stattdessen ein Verhältnis von Vergangenheit und Gegenwart, das durch Kommunikativität, Dialogizität und die „Erschließung eines virtuell synchronen Zeithorizonts“ (A. Assmann 1999a: 65) geprägt ist. Wieder sind es moderne Gesellschaften, in denen diese Potenzialität von Tradition, mit der Gegenwart in einen Austausch zu treten und in gegenwärtigen Deutungsprozessen ihre Sinnstruktur freizugeben, zu voller Entfaltung gebracht wird (vgl. A. Assmann 1999a: 66). Und wieder sind es Schrift und Text, die die reflexive Beziehung zwischen Gegenwart und Vergangenheit in Reinform verkörpern. Dies ist in dem Argument begründet, dass erst das Aufkommen von Schriftlichkeit es ermöglicht, eine Zäsur zwischen Vergangenheit und Gegenwart einzuziehen und damit den vorreflexiv verlaufenden kulturellen Wandel zu einem Verständnis kultureller Entwicklung und Identität aufzustufen. Gerade das Dunkelwerden alter Texte ermöglicht den bewussten Rückgriff auf sie (vgl. Assmann/Assmann 1987: 10). ${ }^{11}$ Von Kopräsenz von Vergangenheit und Gegenwart im Sinne der Assmanns ist daher dann zu sprechen, wenn ein Text der Vergangenheit einem Leser der Gegenwart Ausdrucksformen appräsentiert. Gerade weil der Text zum Lesen gemacht ist, nimmt seine Struktur das Interaktionsverhältnis vorweg, in dem seine späteren Rezeptionen stattfinden werden. Kopräsenz von Gegenwart und Vergangenheit, die die reflexive Verbindung beider begründet, bedeutet somit - Lektüre. Die historische Tiefendimension von Kultur zeigt sich den Assmanns zufolge also in der Prozessstruktur der die Zeit übergreifenden und gleichzeitig auf den Vorgang der Lektüre verdichtenden Interaktion zwischen Leser und kulturellem Text, die in der Struktur von Text und Schrift schon immer angelegt ist.

\section{Rezeption und Kritik}

Über die Arbeiten der Assmanns und ihre Kategorieangebote gibt es eine anhaltende Diskussion in soziologischen, sozialpsychologischen und literaturtheoretischen Kontexten (vg. Dubiel 1999: 243f., Holl 2003, Levy/Sznajder 2001: 43f., Welzer 2002: 13-16, Nünning/Erll 2004). Hier wurde auch Kritik bezüglich der Stringenz und Durchhaltbarkeit der Kategorien geübt. So sei die Unterscheidung zwischen kommunikativem und kulturellen Gedächtnis auf der Ebene der begrifflichen Assoziationen verwirrend, weil die Beschränkung des Adjektivs ,kulturell“ auf außeralltägliche Praktiken einen Anschluss an anthropologische Kulturkonzepte verhindere. Bei derselben Unterscheidung komme es darüber hinaus streckenweise zu einer Identifizierung von Erinnerungsmodi mit zeitlich definierten

11 Hier treffen sich die Assmanns mit kulturanthropologischen Studien, beispielsweise denen Jack Goodys (1981, 2000), die die Möglichkeit eines historischen Bewusstseins im Sinne einer fundamentalen Differenz zwischen Gegenwart und Zukunft an das Aufkommen von Schriftlichkeit und, damit verbunden, der Unterscheidung zwischen Orthodoxie und Heterodoxie binden (s. hierzu auch Langenohl 2007: 58-64). 
Gedächtnisinhalten: dem kulturellen Gedächtnis würden nur diejenigen Repräsentationen zugewiesen, an die sich niemand mehr erinnern könnte (Erll 2005: 113-118). Ferner ist bezweifelt worden, dass das „kulturelle Gedächtnis“ unter den gegenwärtigen Bedingungen abnehmender Verbindlichkeit gepflegter Semantiken noch die Rolle einer normierenden Instanz spielen könne, die die Assmanns ihm beimäßen (Hart 2008: 94).

Diese kritischen Nachfragen zeigen in erster Linie die Produktivität der Assmannschen Unterscheidungen an. Diese sind nicht definitorisch festgelegt, sondern konstituieren sich in ihrer jeweiligen Prägnanz in Gegenüberstellungen mit je unterschiedlichen Gegenkategorien. So stellt die Unterscheidung zwischen kommunikativem und kulturellem Gedächtnis die Aspekte der Institutionalisierung und semantischen Durchformung in den Vordergrund. Dagegen pointiert die Unterscheidung von „kommunikativ“ (Jan) bzw. „sozial“ (Aleida) gegenüber „kollektiv“ (beide) das Unterscheidungskriterium der An- vs. Abwesenheit eines kollektiven imaginaire in der Praxis des Erinnerns. Die Assmannschen Kategorien sind kein fixes Tableau, sondern funktionieren auf der Grundlage von Oppositionspaaren, die je nach Kombination unterschiedliche Eigenschaften und Wirkungen von Erinnerung und Gedächtnis hervorheben (vgl. auch Hart 2008: 93).

\section{Literatur von Jan und Aleida Assmann}

Assmann, A.: Die Legitimität der Fiktion. Ein Beitrag zur Geschichte der literarischen Kommunikation. München 1980.

Assmann, A.: Zur Metaphorik der Erinnerung. In: Assmann, A./Harth, D. (Hg.): Mnemosyne. Formen und Funktionen der kulturellen Erinnerung. Frankfurt/M. 1991, S. 13-35.

Assmann, A.: Arbeit am nationalen Gedächtnis. Eine kurze Geschichte der deutschen Bildungsidee. Frankfurt/New York 1993.

Assmann, A.: Der Eigen-Kommentar als Mittel literarischer Traditionsstiftung. Zu Edmund Spensers The Shepheardes Calendar. In: Assmann, J./Gladikow, B. (Hg.): Text und Kommentar. Archäologie der literarischen Kommunikation IV. München 1995, S. 355-373.

Assmann, A.: Zeit und Tradition. Kulturelle Strategien der Dauer. Köln/Weimar/Wien 1999a.

Assmann, A.: Erinnerungsräume. Formen und Wandlungen des kulturellen Gedächtnisses. München 1999b.

Assmann, A.: Der lange Schatten der Vergangenheit. Erinnerungskultur und Geschichtspolitik. München 2006.

Assmann, A.: Vier Grundtypen von Zeugenschaft. In: Elm, M./Kößler, G. (Hg.): Zeugenschaft des Holocaust. Zwischen Trauma, Tradierung und Erinnerung. Jahrbuch 2007 zur Geschichte und Wirkung des Holocaust. Frankfurt/New York 2007, S. 33-51.

Assmann, A./Assmann, J.: Schrift und Gedächtnis. In: Assmann, A./Assmann, J./Hartmeier, C. (Hg.): Schrift und Gedächtnis. Archäologie der literarischen Kommunikation I. München 1983, S. 265284.

Assmann, A./Assmann, J.: Kanon und Zensur. In: Assmann, A./Assmann, J. (Hg.): Kanon und Zensur. Archäologie der literarischen Kommunikation II. München 1987, S. 7-27.

Assmann, A./Assmann, J.: Das Gestern im Heute. Medien und soziales Gedächtnis. Funkkolleg Medien und Kommunikation. Konstruktionen von Wirklichkeit, Studieneinheit 1, Studienbrief 5. Weinheim/Basel 1990, S. 41-82.

Assmann, A./Assmann, J.: Einleitung. Hieroglyphen: Altägyptische Ursprünge abendländischer Grammatologie. In: Assmann, A./Assmann, J. (Hg.): Hieroglyphen. Stationen einer anderen abendländischen Grammatologie. Archäologie der literarischen Kommunikation VIII. München 2003, S. 9-25. 
Assmann, A./Assmann, J.: Einleitung. In: dies. (Hg.): Verwandlungen. Archäologie der literarischen Kommunikation IX. München 2006, S. 9-24.

Assmann, J.: Die Verborgenheit des Mythos in Ägypten. In: Göttinger Miszellen 25, 1977, S. 1-25.

Assmann, J.: Das kulturelle Gedächtnis. Schrift, Erinnerung und politische Identität in frühen Hochkulturen. München 1992.

Assmann, J.: Text und Kommentar. Einführung. In: Assmann, J./Gladigow, B. (Hg.): Text und Kommentar. Archäologie der literarischen Kommunikation IV. München 1995a, S. 9-33.

Assmann, J.: Erinnern, um dazuzugehören. Kulturelles Gedächtnis, Zugehörigkeitsstruktur und normative Vergangenheit. In: Platt, K./Dabag, M. (Hg.): Generation und Gedächtnis. Erinnerungen und kollektive Identitäten. Opladen 1995b, S. 51-75.

Assmann, J.: Moses der Ägypter. Entzifferung einer Gedächtnisspur. München/Wien 1998.

Assmann, J.: Ägypten: Eine Sinngeschichte. Frankfurt/M. 1999.

Assmann, J.: Religion und kulturelles Gedächtnis. Zehn Studien. München 2000, S. 185-199.

Assmann, J.: Tod und Jenseits im Alten Ägypten. München 2001.

Assmann, J.: Die Zauberflöte. Oper und Mysterium. München/Wien 2005.

Assmann, J.: Erinnertes Ägypten. Pharaonische Motive in der europäischen Religions- und Geistesgeschichte. Berlin 2006.

\section{Weitere zitierte Literatur}

Anderson, B.: Imagined Communities: Reflections on the Origin and Spread of Nationalism. London 1987.

Baer, U.: „Niemand zeugt für den Zeugen“. Erinnerungskultur und historische Verantwortung nach der Shoah. Frankfurt/M. 2000.

Blumenberg, H.: Arbeit am Mythos. Frankfurt/M. 1979.

Diner, D. (Hg.): Zivilisationsbruch. Denken nach Auschwitz. Frankfurt/M. 1988.

Dubiel, H.: Niemand ist frei von der Geschichte. Die nationalsozialistische Herrschaft in den Debatten des Deutschen Bundestages. München/Wien 1999.

Ehlich, K.: Text und sprachliches Handeln. Die Entstehung von Texten aus dem Bedürfnis nach Überlieferung. In: Assmann, A./Assmann, J./Hardmeier, C. (Hg.): Schrift und Gedächtnis. Archäologie der literarischen Kommunikation I. München 1983, S. 24-43.

Erll, A.: Kollektives Gedächtnis und Erinnerungskulturen. Stuttgart/Weimar 2005.

Frank, M. C./Rippl, G.: Arbeit am Gedächtnis. Zur Einführung. In: Frank, M. C./Rippl, G. (Hg.): Arbeit am Gedächtnis. Für Aleida Assmann. München 2007, S. 9-28.

Freud, S.: Der Mann Moses und die monotheistische Religion. Frankfurt/M. 1999 (1939).

Goody, J.: Literalität in traditionalen Gesellschaften. Franfurt a.M. 1981.

Goody, J.: The Power of the Written Tradition. Washington/London 2000.

Gumbrecht, H. U.: Schriftlichkeit in mündlicher Kultur. In: Assmann, A./Assmann, J./Hardmeier, C. (Hg.): Schrift und Gedächtnis. Archäologie der literarischen Kommunikation I. München 1983, S. $185-174$.

Gumbrecht, H. U.: „Phoenix aus der Asche“ oder: Vom Kanon zur Klassik. In: Assmann, A./Assmann, J. (Hg.): Kanon und Zensur. Archäologie der literarischen Kommunikation II. München 1987, S. 284-299.

Halbwachs, M.: Das Gedächtnis und seine sozialen Bedingungen. Berlin/Neuwied 1966 (Ersterscheinen 1925).

Halbwachs, M.: Das kollektive Gedächtnis. Stuttgart 1967.

Harth, D.: The Invention of Cultural Memory. In: Erll, A./Nünning, A. (Hg.): Cultural Memory Studies: An International and Interdisciplinary Handbook. Berlin/New York 2008, S. 85-96.

Holl, M.-K.: Semantik und soziales Gedächtnis. Die Systemtheorie Niklas Luhmanns und die Gedächtnistheorie von Aleida und Jan Assmann. Würzburg 2003. 
Iser, W.: Der implizite Leser. Kommunikationsformen des Romans von Bunyan bis Beckett. München 1972.

Iser, W.: Das Fiktive und das Imaginäre. Perspektiven literarischer Anthropologie. Frankfurt/M. 1991.

Jauß, H. R.: Nachahmung und Illusion. Poetik und Hermeneutik I. München 1964.

Jauß, H. R.: Ästhetische Erfahrung und literarische Hermeneutik. Frankfurt/M. 1982.

Koselleck, R.: Vergangene Zukunft. Zur Semantik geschichtlicher Zeiten. Frankfurt/M. 1989.

LaCapra, D.: History in transit: Experience, Identity, Critical Theory. Ithaca 2004.

Lachmann, R.: Kanon und Gegenkanon in der russischen Kultur des 17. Jahrhunderts. In: Assmann, A./Assmann, J. (Hg.): Kanon und Zensur. Archäologie der literarischen Kommunikation II. München 1987, S: 124-137.

Langenohl, A.: Die Erinnerungsreflexion des Großen Vaterländischen Krieges in Russland, 1995 und 2005. Jahrbuch für historische Kommunismusforschung 2005, S. 68-80.

Langenohl, A. (2007): Tradition und Gesellschaftskritik. Eine Rekonstruktion der Modernisierungstheorie. Frankfurt/M./New York: Campus.

Levy, D./Sznaider, N.: Erinnerung im globalen Zeitalter: Der Holocaust. Frankfurt/M. 2001.

Nora, P.: Between memory and history: Les lieux de mémoire. In: Zemon Davies, N./Starn, R. (Hg.): Memory and counter-memory. Representations 26. Berkeley 1989, S. 7-25.

Luhmann, N. (1997): Die Gesellschaft der Gesellschaft. Erster Teilband. Frankfurt/M. 1997.

Welzer, H.: Das kommunikative Gedächtnis. Eine Theorie der Erinnerung. München 2002.

Wingert, L.: Unpathetisches Ideal. In: Brunkhorst, H. (Hg.): Demokratischer Experimentalismus. Frankfurt/M. 1998, S. 33-43. 